\title{
Electron-electron interactions in graphene sheets
}

\author{
J. González, ${ }^{1}$ F. Guinea, ${ }^{2}$ and M. A. H. Vozmediano ${ }^{3}$ \\ ${ }^{1}$ Instituto de Estructura de la Materia, Consejo Superior de Investigaciones Científicas, Serrano 123, 28006 Madrid, Spain \\ ${ }^{2}$ Instituto de Ciencia de Materiales, Consejo Superior de Investigaciones Científicas, Cantoblanco, 28049 Madrid, Spain \\ ${ }^{3}$ Departamento de Matemáticas, Universidad Carlos III, Butarque 15, Leganés, 28913 Madrid, Spain
}

(Received 12 July 2000; published 7 March 2001)

\begin{abstract}
The effects of the electron-electron interactions in a graphene layer are investigated. It is shown that short-range couplings are irrelevant and scale towards zero at low energies, due to the vanishing of density of states at the Fermi level. Topological disorder enhances the density of states and can lead to instabilities. In the presence of sufficiently strong repulsive interactions, $p$-wave superconductivity can emerge.
\end{abstract}

DOI: 10.1103/PhysRevB.63.134421

PACS number(s): 75.10.Jm, 75.10.Lp, 75.30.Ds

\section{INTRODUCTION.}

Recent experiments ${ }^{1,2}$ report the existence of ferromagnetic and superconducting fluctuations in graphite at unexpectedly high temperatures $(T \sim 100-300 \mathrm{~K})$. The coexistence of both types of fluctuations suggests a common electronic origin for them.

Motivated by these observations, we present here a study of the possible electronic instabilities of a single graphene sheet. Isolated graphene has the convenient property that the electronic states near the Fermi level can be described in simple terms. By symmetry, the lower and upper bands touch at the corners of the hexagonal Brillouin zone. Near these points, the dispersion relation is isotropic and linear, $\epsilon_{\vec{k}}$ $=v_{F}|\overrightarrow{\mathbf{k}}|$, where $v_{F}$ is the Fermi velocity. The density of states at the Fermi level is strictly zero, and it rises linearly in energy. An effective long-wavelength description of these electronic states can be written in terms of the Dirac equation in two dimensions. This description allows us to deal with the effects induced by long- and short-range electronelectron interactions and lattice disorder using standard methods of quantum field theory (see below). While some care must be have when comparing results obtained for this model to real experiments, a single graphene layer provides us with a convenient model which can be used as a starting point for the analysis of many-body effects in graphite. A discussion of the advantages and shortcomings of our study is also included.

The fact that a single graphene sheet is a zero-gap semiconductor modifies significantly the screening of the Coulomb interaction. ${ }^{3}$ An effective low-energy Hamiltonian can be written, which can be treated by renormalization group (RG) methods. ${ }^{4,5}$ It can be shown rigorously that the Coulomb interaction is a marginal interaction, which scales to zero at low energies or long wavelengths. At intermediate scales, however, the quasiparticle lifetime does not follow the usual $\epsilon^{2}$ dependence of Landau's theory of a Fermi liquid, but scales as $|\epsilon|,{ }^{6}$ in agreement with experiments. ${ }^{7}$ The $\mathrm{RG}$ approach is, in principle, valid in the weak coupling regime $\left(e^{2} /\left(\epsilon_{0} v_{F}\right) \ll 1\right.$, where $e$ is the electric charge and $\epsilon_{0}$ is the contribution to the dielectric constant from the $\sigma$ orbitals and core electrons. By using a random phase approximation (RPA) summation of diagrams, it can be shown that the low energy properties are not changed throughout the entire range of couplings. ${ }^{8}$

The previous work mentioned earlier analyzed the small momentum scattering due to the long-range Coulomb interaction, as it is the only one which leads to logarithmically divergent perturbative corrections. Some electronic instabilities, like anisotropic superconductivity, require the existence of short-range interactions with significant strength at finite wave vectors. We analyze in this work the role of these interactions in inducing instabilities of the electronic system. The next section describes the model. Then, the renormalization group equations for the different interactions are written. In Sec. IV, the role of topological disorder is analyzed, as it can lead to changes in the density of states which modify the scaling equations obtained earlier. Section V discusses effects not included in the model and how they can influence the results presented so far. In particular, we analyze the changes induced by the hopping between neighboring graphene layers. Section VI presents the expected main features of the low-temperature instabilities of a graphene layer and discusses its experimental implications.

\section{MODEL}

\section{A. Intralayer couplings}

We analyze the low-energy properties of a graphene sheet. We will only consider the modifications due to interactions and disorder in the low-energy properties of the sys-

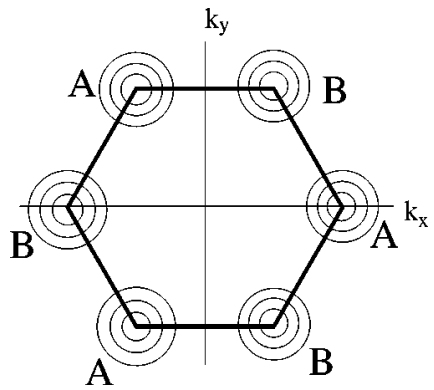

FIG. 1. Sketch of the Brillouin zone of a graphene sheet and the band dispersion near the Fermi energy. Points A and B refer to the two set of points which are related between themselves by a reciprocal vector translation. 
tem. Thus, we need to describe the low-energy electronic states. A graphene sheet has an hexagonal symmetry with two atoms per unit cell. The carbon atoms have four valence orbitals. Three of them build the $s p^{2}$ bonds which give rigidity to the structure. The third orbital gives rise to the valence and conduction bands. These bands touch at the two inequivalent corners of the Brillouin zone (see Fig. 1). From symmetry considerations, these bands are isotropic and depend linearly on the wave vector.

It can be shown that, in the long-wavelength limit, the electronic wave functions near the corners of the Brillouin zone are well described in terms of the two-dimensional Dirac equation. The six corners of the Brillouin zone can be divided into two inequivalent sets A and B (see Fig. 1). The three corners with the same label are related by reciprocal lattice vectors and can be considered a single point. Thus, a long-wavelength description requires two Dirac spinors, each of them with its spin index. We refer to the $\overrightarrow{\mathbf{k}}$ point index $\mathrm{A}(\mathrm{B})$ as the flavor index following standard procedures. In the long-wavelength limit, the Fermi velocity $v_{F}$ can be expressed in terms of the matrix elements between nearestneighbor $\pi$ orbitals, $t$, as $v_{F}=(3 t a) / 2$, where $a$ is the C-C distance.

Because of the collapse of the Fermi surface to isolated points, the kinematics are much simpler than the corresponding analysis for two "hot spots" in a square lattice. ${ }^{9}$ The Hamiltonian is

$$
\begin{aligned}
\mathcal{H}= & \sum_{i, s} \hbar v_{F} \int d^{2} r \bar{\Psi}_{i, s}(\vec{r})\left(i \sigma_{x} \partial_{x}+i \sigma_{y} \partial_{y}\right) \Psi_{i, s}(\vec{r})+\sum_{i, i^{\prime} ; s, s^{\prime}} \frac{e^{2}}{2 \epsilon_{0}} \int d^{2} r_{1} \int d^{2} r_{2} \frac{\bar{\Psi}_{i, s}\left(\vec{r}_{1}\right) \Psi_{i, s}\left(\vec{r}_{1}\right) \bar{\Psi}_{i^{\prime}, s^{\prime}}\left(\vec{r}_{2}\right) \Psi_{i^{\prime}, s^{\prime}}\left(\vec{r}_{2}\right)}{\left|\vec{r}_{1}-\vec{r}_{2}\right|} \\
& +\sum_{s, s^{\prime} ; i, i^{\prime}} g_{i, s ; i^{\prime}, s^{\prime}} \int d^{2} r \bar{\Psi}_{i, s}(\vec{r}) \Psi_{i, s}(\vec{r}) \bar{\Psi}_{i^{\prime}, s^{\prime}}(\vec{r}) \Psi_{i^{\prime}, s^{\prime}}(\vec{r}) \\
& +\sum_{s, s^{\prime} ; i, i^{\prime}} \bar{g}_{i, s ; i^{\prime}, s^{\prime}} \int d^{2} r \bar{\Psi}_{i, s}(\vec{r}) \vec{\sigma} \Psi_{i, s}(\vec{r}) \bar{\Psi}_{i^{\prime}, s^{\prime}}(\vec{r}) \vec{\sigma} \Psi_{i^{\prime}, s^{\prime}}(\vec{r})
\end{aligned}
$$

where $\sigma_{x}$ and $\sigma_{y}$ are $2 \times 2$ Pauli matrices. We have separated the long-wavelength part of the Coulomb interaction from other possible short-range interactions.

The couplings $g_{i, i^{\prime} ; s, s^{\prime}}$ can be classified in an analogous way as in one dimension. The possible scattering processes are shown in Fig. 2.

Because of the linear dispersion of the electronic states, we can use $v_{F}$ to transform time scales into length scales. Then, we can express the dimensions of all physical quantities in terms of lengths. Within this convention, we find that the dimension of the electronic fields is $[\Psi]=l^{-1}$, where $l$ defines a length. A naive power counting analysis shows that the Coulomb potential defines a dimensionless, marginal coupling, while the $g$ 's scale as $l$ and are irrelevant at low energies. This effect can be traced back to the vanishing

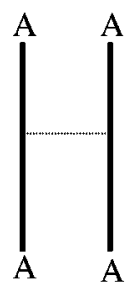

a)

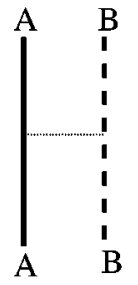

b)

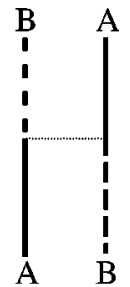

c)
FIG. 2. Possible short-range couplings between electrons near the two Fermi points in a graphene layer. The solid and dashed lines correspond to electrons in the vicinity of each of the two Fermi points. (a) Intrasingularity scattering $\left(g_{\text {intra }}\right)$. (b) Intersingularity scattering $\left(g_{\text {inter }}\right)$. (c) Exchange scattering $\left(g_{\text {exchange }}\right)$. density of states at the Fermi level. When a single Hubbard intrasite repulsion $U$ is considered, all interactions between electrons of opposite spin in Eq. (1) are equal to $U \Omega$, where $\Omega$ is the area of the unit cell, and the interactions between electrons of parallel spin are zero.

\section{B. Interlayer couplings}

So far, we have restricted our analysis to processes within an isolated graphene sheet. Neighboring layers are always coupled by the Coulomb interaction. In the following, we will neglect interlayer hopping, so as to be able to describe the electronic levels in terms of the Dirac equation, but we include the effects of the long-range Coulomb interactions between layers. The interlayer couplings give rise to the screening of the bare intralayer electron-electron interaction. We will treat these effects within the RPA, as depicted in Fig. 3, following the analysis in Ref. 6. The intralayer inter-

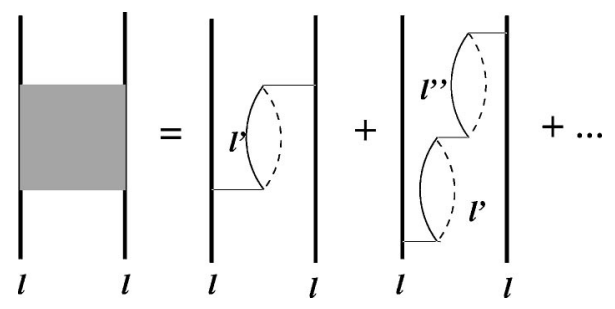

FIG. 3. Diagrammatic representation of the random phase approximation applied to the interlayer Coulomb interaction. The diagrams show the screening of the interaction between two electrons in layer $l$ due to the polarization of layers $l^{\prime}, l^{\prime \prime}, \ldots$. 
action becomes

$$
v_{s c r}(\omega, \overrightarrow{\mathbf{q}})=\frac{2 \pi e^{2}}{\epsilon_{0}|\overrightarrow{\mathbf{q}}|} \frac{\sinh (|\overrightarrow{\mathbf{q}}| d)}{\sqrt{\left[\cosh (|\overrightarrow{\mathbf{q}}| d)+\left(2 \pi e^{2} / \boldsymbol{\epsilon}_{0}|\overrightarrow{\mathbf{q}}|\right) \sinh (|\overrightarrow{\mathbf{q}}| d) \chi_{0}(\omega, \overrightarrow{\mathbf{q}})\right]^{2}-1}}
$$

where $d$ is the distance between layers, and $\chi_{0}$ is the electron susceptibility of a single layer, given by

$$
\chi_{0}(\omega, \overrightarrow{\mathbf{q}})=\frac{\overrightarrow{\mathbf{q}}^{2}}{32 \pi \sqrt{v_{F}^{2} \overrightarrow{\mathbf{q}}^{2}-\omega^{2}}}
$$

The interlayer interactions are only effective when $|\overrightarrow{\mathbf{q}}| d$ $\ll 1$. Hence, if the lattice constant $a$ is such that $a \ll d$, they do not affect significantly the couplings between electronic states in different Fermi points.

\section{SCALING ANALYSIS}

In Ref. 8 it was shown that the electrostatic coupling, defined as $e^{2} /\left(\epsilon_{0} v_{F}\right)$, scales towards zero at low energies, for all values of the interaction. On the other hand, the existence of scattering processes between the two inequivalent Fermi points can lead to instabilities at intermediate couplings. Different combinations of couplings lead to each instability. The system becomes ferromagnetic for sufficiently large values of $g_{\text {intra } \perp}+g_{\text {inter } \perp}-g_{\text {intra } \|}-g_{\text {inter } \|}$, where the subscripts $\|$ and $\perp$ denote the relative orientation between spins. An antiferromagnetic instability is driven by $\bar{g}_{\text {intra } \perp}$ $+\bar{g}_{\text {inter } \perp}-\bar{g}_{\text {intra } \|}-\bar{g}_{\text {inter } \|}$. The superconducting phases can be $s$ and $p$ wave, depending on the relative phase of the gap at the two inequivalent points. However, for each $\overrightarrow{\mathbf{k}}$ near the Fermi points, there are two electronic states, so that an additional index can be defined in the superconducting order parameter. Writing these two states as a two component spinor, we can write, in general,

$$
\Delta_{\overrightarrow{\mathbf{k}}}=\left\langle\Psi_{A, \uparrow, \overrightarrow{\mathbf{k}}}(a \mathcal{I}+\overrightarrow{\mathbf{b}} \vec{\sigma}) \Psi_{B, \downarrow,-\overrightarrow{\mathbf{k}}}\right\rangle
$$

where $a$ and $\overrightarrow{\mathbf{b}}$ are constants. When the interaction is repulsive, the $p$-wave symmetry is favored $\left(\Delta_{\overrightarrow{\mathbf{k}}}=-\Delta_{-\overrightarrow{\mathbf{k}}}\right)$, as in a two-dimensional (2D) electron system with two inequivalent Van Hove singularities at the Fermi level. ${ }^{9}$ The corresponding coupling is $g_{\text {inter } \perp}+\bar{g}_{\text {inter } \perp}-g_{\text {exchange } \perp}-\bar{g}_{\text {exchange } \perp \text {. The }}$ diagrams which define the flow of these couplings are depicted in Fig. 4.

The corresponding equations for the dimensionless vertices $\Gamma^{\prime}$ 's can be written as

$$
\begin{gathered}
\frac{\partial \widetilde{\Gamma}_{\text {inter }}}{\partial \ln (\Lambda)}=-d_{\widetilde{\Gamma}} \widetilde{\Gamma}_{\text {inter }}-\widetilde{\Gamma}_{\text {inter }}^{2}-\widetilde{\Gamma}_{\text {exchange }}^{2}, \\
\frac{\partial \widetilde{\Gamma}_{\text {exchange }}}{\partial \ln (\Lambda)}=-d_{\widetilde{\Gamma}} \widetilde{\Gamma}_{\text {exchange }}-2 \widetilde{\Gamma}_{\text {exchange }} \widetilde{\Gamma}_{\text {inter }},
\end{gathered}
$$

where we are omitting spin and flavor indices for simplicity, and $d_{\widetilde{\Gamma}}$ is the (anomalous) dimension of the vortex, which includes, among others, the effects of the wave function renormalization of the fields. To lowest order, $d_{\widetilde{\Gamma}}=1$. The first term on the right-hand sides of Eqs. (5) is linear, and it is absent in the flow of the couplings in the Cooper channel in a conventional metal. It reflects the irrelevance of these couplings in a semimetal.

The flow in this channel becomes relevant if $\widetilde{\Gamma}_{\text {exchange }}$ $\geqslant \widetilde{\Gamma}_{\text {inter }}$ and the values of the $\widetilde{\Gamma}^{\prime}$ 's are of order unity. Note that the cutoff is assumed to be $\Lambda \approx v_{F} / a$, where $a$ is a length of the order of the lattice constant. The dimensionful interFermi points and exchange couplings induced by the Coulomb interactions are $g_{i} \sim e^{2} /\left(\epsilon_{0} a\right)$. Hence, the bare vortices $\widetilde{\Gamma}_{0} \sim e^{2} /\left(\epsilon_{0} v_{F}\right)$. For reasonable values of $\epsilon_{0} \sim 4-8$, this combination is, indeed, of order unity.

\section{INFLUENCE OF DISORDER}

\section{A. Topological disorder}

The formation of pentagons and heptagons in the lattice, without affecting the threefold coordination of the carbon atoms, leads to the warping of the graphene sheets and is responsible for the formation of curved fullerenes, like $C_{60}$. They have also been observed in carbon nanotubes. In graphene, the number of pentagon defects should equal the number of heptagons, in order to preserve the flatness at large scales of the sheets. Pentagons and heptagons can be viewed as disclinations in the lattice, and, when circling one such defect, the two sublattices in the honeycomb structure are exchanged (see Fig. 5).

The two fermion flavors defined in Eq. (1) are also exchanged when moving around such a defect. The scheme to incorporate this change in a continuum description was discussed in Ref. 10. The process can be described by means of a non-Abelian gauge field, which rotates the spinors in flavor space. The vector potential is that of a vortex at the position of the defect, and the flux is $\pm \pi / 2$.

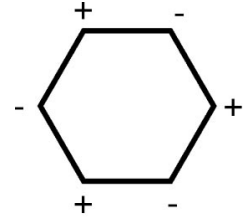

a)

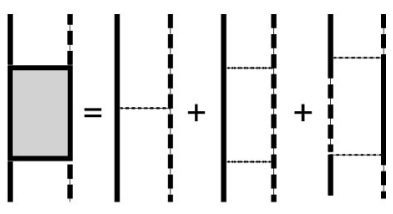

b)
FIG. 4. Renormalization group equations in the Cooper channel with $p$-wave symmetry. (a) Sketch of the order parameter in the Brillouin zone. (b) Diagrams involved in the calculation. 


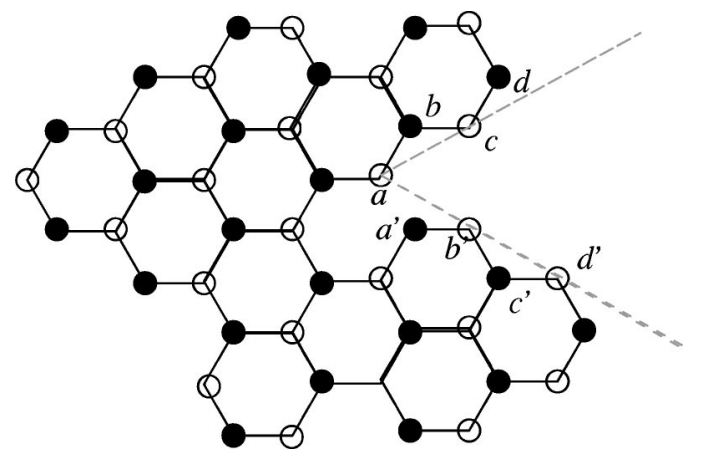

FIG. 5. Formation of a pentagonal ring in the honeycomb lattice. Points $a, b, c, d, \ldots$ have to be identified with points $a^{\prime}, b^{\prime}, c^{\prime}, d^{\prime}, \ldots$. The defect can be seen as a disclination, defined by the straight dashed lines.

Dislocations can be analyzed in terms of bound disclinations, that is, a pentagon and an heptagon located at short distances, which define the Burgers vector of the dislocation. Thus, the effect of a dislocation on the electronic levels of a graphene sheet is analogous to that of the vector potential arising from a vortex-antivortex pair. We can extend this description ${ }^{11}$ and assume that a lattice distortion which rotates the lattice axis can be parametrized by the angle of rotation, $\theta(\overrightarrow{\mathbf{r}})$, of the local axes with respect to a fixed reference frame. Then, this distortion induces a gauge field such that

$$
\overrightarrow{\mathbf{A}}(\overrightarrow{\mathbf{r}})=3 \nabla \theta(\overrightarrow{\mathbf{r}})\left(\begin{array}{cc}
0 & -i \\
i & 0
\end{array}\right)
$$

Thus, a random distribution of topological defects can be described by a (non-Abelian) random gauge field. The nature of the electronic states derived from the two-dimensional Dirac equation in the presence of a gauge field with Gaussian randomness has received a great deal of attention, as it also describes the effects of disorder in integer quantum Hall transitions. ${ }^{12}$ The disorder is defined by a single dimensionless quantity $\Delta$, which is proportional to the average fluctuations of the field:

$$
\left\langle\overrightarrow{\mathbf{A}}(\overrightarrow{\mathbf{r}}) \overrightarrow{\mathbf{A}}\left(\overrightarrow{\mathbf{r}}^{\prime}\right)\right\rangle=\Delta \delta^{2}\left(\overrightarrow{\mathbf{r}}-\overrightarrow{\mathbf{r}}^{\prime}\right) .
$$

It is known that $\Delta$ gives rise to a marginal perturbation, which modifies the dimensions of the fermion fields and enhances the density of states at low energies. A variety of analytical ${ }^{15}$ and numerical techniques ${ }^{16}$ has been used to study this problem. We will follow the renormalization group scheme presented in Ref. 12.

We first analyze the statistical properties of the gauge field induced by topological defects. Let us assume that the graphene sheet is warped and that there is a random distribution of pentagons and heptagons with density $n_{0}$ and average distance equal to $l_{0}=n_{0}^{-1 / 2}$. The fluctuations in the gauge field induced by this distribution at a given point can be calculated by considering the effect of all defects located at distances between $r$ and $r+d r$ (see Fig. 6), where $r \gg l_{0}$. The number of defects of each type is $2 \pi r d r n_{0}$. The angle

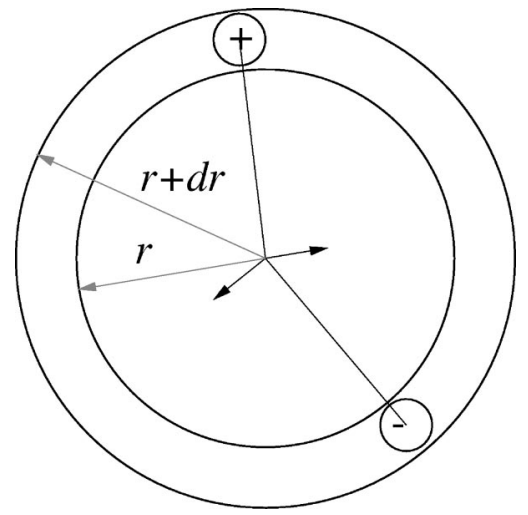

FIG. 6. Effect of vortices located at distances between $r$ and $r$ $+d r$ from the origin (see text for discussion).

$\phi$ which defines their position is a random variable. The contribution to the $x$ component of the gauge field from these vortices is

$$
A_{x}^{2}(\overrightarrow{\mathbf{r}}=0)=\left(\frac{\Phi_{0}}{r}\right)^{2}\left[\sum_{i} \cos \left(\theta_{i}\right)\right]^{2}=\left(\frac{\Phi_{0}}{r}\right)^{2} \frac{1}{2} 2 \pi n_{0} r d r
$$

where $\Phi_{0}$ is the flux associated with a single vortex, and there is a similar equation for $A_{y}(\overrightarrow{\mathbf{r}}=0)$. We now must integrate this value from $l_{0}$ to $R$, where $R$ is the radius of the sample. We obtain

$$
|\overrightarrow{\mathbf{A}}(\overrightarrow{\mathbf{r}}=0)|^{2}=2 \pi n_{0} \Phi_{0}^{2} \ln \left(\frac{R}{l_{0}}\right) .
$$

We can assume that the vector potential at positions separated by distances greater than $l_{0}$ are not correlated. Then, from Eq. (7), we find

$$
\Delta=2 \pi \Phi_{0}^{2} \ln \left(\frac{R}{l_{0}}\right),
$$

which diverges slowly with the size of the system. The previous estimate assumed that the layers had a significant amount of curvature at distances smaller than $l_{0}$. We can alternatively assume that pentagons and heptagons are bound in dislocations with average distance $b$. The vector field of a vortex-antivortex dipole decays as $r^{-2}$. A similar analysis to the one leading to Eq. (10) gives

$$
\Delta \propto \Phi_{0}^{2} n_{\text {disl }} b^{2},
$$

where $n_{\text {disl }}$ is the density of dislocations.

We will now assume that random fields induced by topological defects have the same statistical properties to those with Gaussian disorder with the same value of $\Delta$, which is the second moment of the distribution in both cases. Then, we can perform the renormalization group analysis discussed in Ref. 12. To lowest order, we find an interaction between fermion fields in different replicas of the type 


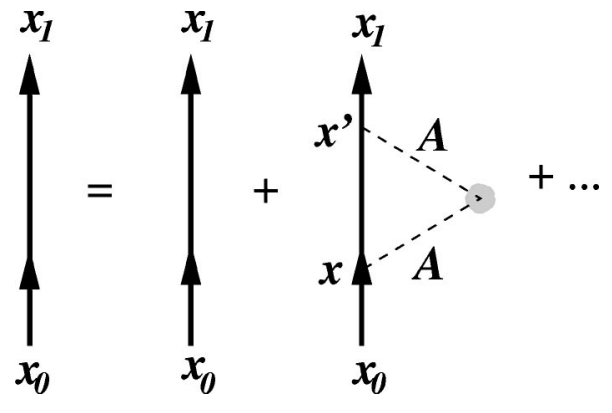

FIG. 7. Diagrams contributing to the Green's function in a disordered electron system.

$$
\begin{aligned}
S_{i n t}= & \Delta \sum_{m, n} \int\left[\bar{\Psi}_{A}\left(\overrightarrow{\mathbf{r}}, t_{1}\right) \Psi_{B}\left(\overrightarrow{\mathbf{r}}, t_{1}\right)\right]_{m} \\
& \times\left[\bar{\Psi}_{B}\left(\overrightarrow{\mathbf{r}}, t_{2}\right) \Psi_{A}\left(\overrightarrow{\mathbf{r}}, t_{2}\right)\right]_{n} d t_{1} d t_{2} d \overrightarrow{\mathbf{r}},
\end{aligned}
$$

where $m$ and $n$ are replica indices. This interaction leads to a logarithmically divergent self-energy, which can be interpreted as a renormalization of the density of states. ${ }^{12}$ We can include the corrections induced by the self-energy in a renormalization of the wave function, giving rise to a change in the scaling dimension of the fields:

$$
2 d_{\Psi}-1=1-\frac{\Delta}{\pi}
$$

This expression has to be inserted in Eq. (5), modifying the flow of the couplings.

The same result can be reached by analyzing the selfenergy corrections using standard techniques in the study of disordered electrons in arbitrary dimensions. ${ }^{13}$ To lowest order, the first correction to the Green's function is shown in Fig. 7. This diagram leads to a self-energy

$$
\begin{aligned}
\Sigma\left(\overrightarrow{\mathbf{r}}, \overrightarrow{\mathbf{r}}^{\prime}, \omega\right) & \approx\left\langle G_{0}\left(\overrightarrow{\mathbf{r}}-\overrightarrow{\mathbf{r}}^{\prime}, \omega\right) \overrightarrow{\mathbf{A}}(\overrightarrow{\mathbf{r}}) \overrightarrow{\mathbf{A}}\left(\overrightarrow{\mathbf{r}}^{\prime}\right)\right\rangle+\cdots \\
& =\Delta G_{0}\left(\overrightarrow{\mathbf{r}}-\overrightarrow{\mathbf{r}}^{\prime}, \omega\right) \delta^{2}\left(\overrightarrow{\mathbf{r}}-\overrightarrow{\mathbf{r}}^{\prime}\right)+\cdots,
\end{aligned}
$$

where $G_{0}$ is the unperturbed Green's function. The real part of $G_{0}$ behaves as $G_{0} \sim \omega \ln (\Lambda / \omega)$. Finally,

$$
2 d_{\Psi}-1=1-\frac{\partial}{\partial \ln \Lambda}\left(\frac{\partial \Sigma}{\partial \omega}\right) .
$$

The previous perturbative analysis can be generalized to arbitrary couplings, by mapping the noninteracting fermion problem in two spatial dimensions onto an interacting problem in $1+1$ dimensions. ${ }^{14}$ At energies below a scale $\lambda$ $\sim \Lambda \exp [-\pi /(2 \Delta)]$, the backscattering between the two Fermi points leads to a scaling dimension which is independent of the disorder, $d_{\Psi}=1 / 7$.

\section{B. Substitutional and site disorder}

It can be shown that substitutional and site disorder can be incorporated into the Dirac equation through a change in the local chemical potential and the appearance of a mass term. ${ }^{15}$ Disorder of these types, with a Gaussian distribution, defines a marginally relevant perturbation. ${ }^{15}$ Within the perturbative RG scheme described in the previous subsection, it can be shown that this perturbation leads to logarithmic corrections to the site-diagonal self-energy, which can be incorporated into a renormalization of the wave function. Moreover, deviations from a Gaussian distribution are relevant perturbations, which modify the results.

Thus, while to lowest order substitutional disorder shows similar characteristics as topological disorder, higher-order corrections lead to significant modifications, which, in addition, depend on the type of disorder. ${ }^{17}$ For instance, a sharp divergence of the density of states has been found ${ }^{18}$ or a suppression of the density of states at low energies. ${ }^{19}$

In graphite, we do not expect a high concentration of charged impurities, which will lead to a strong site disorder. Randomness in bond lengths leads to nondiagonal disorder, which can be included in the topological disorder discussed in the previous subsection. Hence, we expect diagonal disorder in pure graphite to be small, leading to minor corrections to the dependence of the density of states on energy, even when nonperturbative terms are included. ${ }^{17}$

\section{OTHER EFFECTS NOT INCLUDED IN THE MODEL}

\section{A. Phonons}

It is well known that phonons can induce significant effects in conjugated systems, such as poliacetylene, ${ }^{20}$ and in graphite intercalation compounds. ${ }^{21,22}$ There is no evidence, however, for a similar charge (or bond) density wave in graphite, where a 1D Peierls instability is absent. Phonons play an important role in $\mathrm{C}_{60}$ compounds, ${ }^{23}$ where they may be responsible for the superconductivity in some $A_{3} \mathrm{C}_{60}$ materials. The relevance of the electron-phonon coupling is due to the existence of very narrow electronic bands, due to the weak hybridization of $\mathrm{C}_{60}$ molecules, and quasilocalized phonon modes. Neither of these features can be extended to graphite. Finally, our main purpose is the study of instabilities towards ground states which exhibit magnetism or anisotropic superconductivity. We assume that the electronphonon interaction will not change qualitatively the possible existence of these instabilities. Thus, we do not expect that the inclusion of the electron-phonon coupling will alter significantly the results presented here.

\section{B. Interlayer hopping}

We have also not consider the interlayer hopping $t_{\perp}$. Within the RG scheme, coherent interlayer hopping is a relevant perturbation, ${ }^{24}$ leading to three-dimensional behavior at low energies or temperatures. On the other hand, due to the vanishing of the density of states of a graphene layer, incoherent hopping between layers is irrelevant (note that it is a marginal perturbation in systems with a finite density of states $^{25}$ ).

In the presence of coherent interlayer hopping, our analysis is valid only at scales higher than $t_{\perp}$, which has been estimated, by band structure calculations, to be $t_{\perp}$ $\approx 0.27 \mathrm{eV}^{26}$ This bare value will be reduced by the manybody effects and the wave function renormalization consid- 
ered here. However, in a perfect system, the validity of our calculations are limited to a range between $t \approx 2.4 \mathrm{eV}$ and the renormalized value of $t_{\perp}$.

The coherent interlayer hopping can modify our results in various ways: (i) The coupling between layers induces a crossover to $3 \mathrm{D}$ behavior, enhancing the $2 \mathrm{D}$ instabilities discussed here. (ii) The dispersion of the electronic bands in the third dimension leads to the existence of small electron and hole pockets, increasing the density of states. If the couplings are not modified, this finite density of states will also strengthen the instabilities. (iii) The density of states at the Fermi level induces metallic screening, and changes the interactions at low energies. It is unclear to us how our results are modified in case (iii).

Our calculations have a wider range of validity in the presence of disorder, where coherent hopping over distances longer than the electronic mean free path is suppressed. As mentioned earlier, incoherent local hopping can be considered an irrelevant perturbation which should not modify qualitatively the results presented here.

\section{MAIN FEATURES OF THE LOW-TEMPERATURE PHASE DIAGRAM}

\section{A. Estimates of the couplings}

Our analysis considers the role of electron-electron interactions in a graphene layer. Spin-dependent interactions, like a Hubbard on site term, naturally lead to magnetic phases. In the absence of disorder, a minimum value for the Hubbard repulsion is required before the onset of antiferromagnetism, ${ }^{27}$ in agreement with the analysis presented here. This phase, however, lacks experimental confirmation. It is also known that, within the Hartree-Fock approximation, a nearest-neighbor repulsion $V$ induces a charge-density-wave ground state if $U-3 V<0$ and $U$ and $V$ are sufficiently large. ${ }^{28-30} \mathrm{In}$ addition, there is a region in the phase diagram where $U$ and $V$ almost cancel, leading to a paramagnetic ground state. Realistic values of these parameters suggest that a graphene layer lies in this region. ${ }^{28-30}$ It is reasonable that longer-range correlations can make this state unstable. These calculations do not consider longerrange interactions. For decoupled graphene layers, the vanishing of the density of states at the Fermi level leads to the absence of metallic screening, so that spin-independent, long-range interactions are expected.

\section{B. Possible low-temperature phases}

We have considered the possibility of ferro- and antiferomagnetism and $p$-wave superconductivity as the most likely low-temperature phases. The competition between them depends on the spin dependence of the interactions. Spinindependent couplings favor superconductivity, while a strong spin dependence, like the on-site repulsion of the Hubbard model, will lead to a magnetic ground state. Finally, the relative stability of ferro- and antiferromagnetism depends, among other things, on the existence of an underlying bipartite lattice. In the presence of a sufficiently strong topological disorder, we expect that ferromagnetism will pre- vail over antiferromagnetism, as the existence of pentagons and heptagons leads to the frustration of antiferromagnetic order. The same argument can be applied to the charge density wave state considered in Refs. 28 and 29.

A detailed study of the competition between ferromagnetism and $p$-wave superconductivity lies beyond the scope of this work. It depends on the balance between the on-site, spin-dependent interactions, and the longer-range, spinindependent couplings. Ferromagnetism is favored by the existence of a sufficiently strong forward scattering between electrons of opposite spin, at momentum transfer $\overrightarrow{\mathbf{q}} \approx 0$. This coupling depends on the nature of the screening, which, in turn, depends on the density of states near the Fermi level and on the degree of disorder. On the other hand, if the main interactions are spin independent, ferromagnetism will be suppressed, and the leading instability is $p$-wave superconductivity.

\section{General features of the possible superconducting instability}

In the following, we will discuss some qualitative features of the superconducting transition. A quantitative estimate of the critical temperature is beyond the scope of our RG scheme, although we can discuss the dependence of $T_{c}$ on various quantities.

It is first interesting to note that superconductivity at low temperatures was observed in graphite intercalation compounds. ${ }^{31}$ The origin of this superconductivity is not completely understood. The critical field shows an anomalous dependence on temperature, ${ }^{32}$ unlike in conventional s-wave superconductors. This dependence has been explained in terms of a two-band model..$^{33}$ This model is similar to the two-point model discussed here, except that the two bands considered in Ref. 33 correspond to a carbon and a dopand band. The temperature dependence of the critical field should be, however, similar in the two cases.

The critical temperature at which an instability described by Eq. (5) sets in is

$$
T_{c}=\Lambda\left(\frac{\widetilde{\Gamma}_{0}-d_{\tilde{\Gamma}}}{\widetilde{\Gamma}_{0}}\right)^{1 / d \tilde{\Gamma}},
$$

where $\Gamma$ is the appropriate vortex required to drive the instability. There is a transition if $\widetilde{\Gamma}_{0}>\widetilde{\Gamma}_{c}=d_{\tilde{\Gamma}}$. For $d_{\tilde{\Gamma}}=0$, this expression reduces to the usual BCS formula $T_{c}=\Lambda \exp$ $\left(-1 / \widetilde{\Gamma}_{0}\right)$ and $\widetilde{\Gamma}_{c}=0$. The disorder influences the scaling of the fermion fields $d_{\Psi}$, which, in turn, modify $d_{\tilde{\Gamma}}$ :

$$
d_{\widetilde{\Gamma}}=4 d_{\Psi}-3=1-\frac{2 \Delta}{\pi},
$$

where $\Delta$ is given in Eq. (10) or (11). The critical temperature depends exponentially on the disorder. The expression in Eq. (16) is only valid if $T_{c} \ll v_{F} / d$, where $d$ is the typical distance above which Eq. (10) or (11) holds.

We can make a simple estimate of the role of disorder by assuming that, for certain average separation between de- 
fects, $l_{0}, d_{\widetilde{\Gamma}}=0$, and the value of the critical temperature is $T_{c}^{\max }$. Then, if the disorder is reduced, we expand on $d_{\tilde{g}}$ $\ll 1$, and we obtain

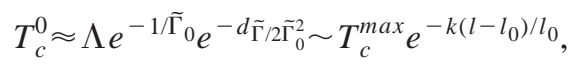

where $k \propto \widetilde{\Gamma}_{0}^{-2}$ is a numerical constant and $l$ is the average distance between defects. The superscript 0 stands for the fact that frustration effects in the superconducting phase are not considered (see below). Finally, we can get a rough estimate for $l_{0}$ by considering that a sufficiently large concentration of defects leads to pair breaking and reduces $T_{c}$ in an anisotropic superconductor. The reduction of $T_{c}$ is given, approximately, by ${ }^{11}$

$$
T_{c} \approx T_{c}^{0}\left(1-c \frac{\xi_{0}^{2}}{l_{0}^{2}}\right) .
$$

where $\xi_{0}=v_{F} / T_{c}^{0}$ is the coherence length of the superconductor, and $c$ is a constant of order unity. Hence, the optimal concentration of defects will be in the range $l_{0} \sim \xi_{0}$. Assuming that $T_{c}^{\max } \sim 300 \mathrm{~K}$, this estimate gives for the mean distance between defects $l_{0} \sim 30-100 \AA$.

\section{Expected sources of disorder in graphene sheets}

It is known that electronic properties of graphite, like the resistivity, are sample dependent, ${ }^{34}$ and localization effects due to disorder have been observed. ${ }^{35}$ As discussed in Sec. IV, the effect of topological disorder depends on whether the graphene sheets present a finite density of disclinations, leading to corrugated and warped surfaces, or the main source of disorder is due to dislocations. Aggragations of graphite nanoparticles of polyhedron shapes, whose curvature is not completely characterized, are discussed in Ref. 36. Warped layers, with curved regions which are reminiscent of the spherical fullerenes, have been observed. ${ }^{37}$ These structures seem similar to proposed models of negatively curved graphene layers. ${ }^{38}$ Theoretically, these compounds (schwarzites) are supposed to be very stable and contain a macroscopic fraction of heptagonal rings. A material with these characteristics is probably best described by a random distribution of disclinations, with mean separation equal to a few lattice spacings. Calculations of the electronic density of states of the model proposed in Ref. 38 show that it loses the semimetallic properties of graphite, in agreement with the discussion here. ${ }^{39}$ Compounds with these characteristics can be good candidates for intrinsic p-wave superconductivity.

From the difference between Eqs. (10) and (11), it is clear that a noncorrugated graphene sheet has a much lower density of states than a significantly warped one and a reduced tendency towards electronic instabilities. In highly disordered graphite, however, it is possible that regions with different degrees of corrugation coexist giving rise to the behavior reported in Refs 1 and 2.

\section{ACKNOWLEDGMENTS}

We are thankful to M. P. López-Sancho, F. Batallán, and P. Esquinazi for helpful discussions. Financial support from MEC (Spain) through Grant No. PB96/0875 and CAM (Madrid) through Grant No. 07/0045/98 is gratefully acknowledged.
${ }^{1}$ Y. Kopelevich, P. Esquinazi, J. H. S. Torres, and S. Moehlecke, J. Low Temp. Phys. 119, 691 (2000).

${ }^{2}$ H. Kempa, Y. Kopelevich, F. Mrowka, A. Setzer, J. H. S. Torres, R. Höhne, and P. Esquinazi, Solid State Commun. 115, 539 (2000)

${ }^{3}$ J. González, F. Guinea, and M. A. H. Vozmediano, Mod. Phys. Lett. B 7, 1593 (1994); Nucl. Phys. B 424, 595 (1994); J. Low Temp. Phys. 99, 287 (1995).

${ }^{4}$ R. Shankar, Rev. Mod. Phys. 66, 129 (1994).

${ }^{5}$ J. Polchinski, in Proceedings of the 1992 TASI in Elementary Particle Physics, edited by J. Harvey and J. Polchinski (World Scientific, Singapore, 1992).

${ }^{6}$ J. González, F. Guinea, and M. A. H. Vozmediano, Phys. Rev. Lett. 77, 3589 (1996).

${ }^{7}$ S. Yu, J. Cao, C. C. Miller, D. A. Mantell, R. J. D. Miller, and Y. Gao, Phys. Rev. Lett. 76, 483 (1996).

${ }^{8}$ J. González, F. Guinea, and M. A. H. Vozmediano, Phys. Rev. B 59, R2474 (1999).

${ }^{9}$ J. González, F. Guinea, and M. A. H. Vozmediano, Phys. Rev. Lett. 84, 4930 (2000).

${ }^{10}$ J. González, F. Guinea, and M. A. H. Vozmediano, Phys. Rev. Lett. 69, 172 (1992); Nucl. Phys. B 406, 771 (1993).

${ }^{11}$ F. Guinea, Phys. Rev. B 58, 6622 (1998).
${ }^{12}$ A. W. W. Ludwig, M. P. A. Fisher, R. Shankar, and G. Grinstein, Phys. Rev. B 50, 7526 (1994).

${ }^{13}$ E. N. Economou, Green's Functions in Quantum Physics (Springer, Berlin, 1983).

${ }^{14}$ A. A. Nersesyan, A. M. Tsvelik, and F. Wenger, Phys. Rev. Lett. 72, 2628 (1994); Nucl. Phys. B 438, 561 (1995).

${ }^{15}$ C. de C. Chamon, C. Mudry, and X. G. Wen, Phys. Rev. Lett. 77, 4194 (1996); Nucl. Phys. B466, 383 (1996).

${ }^{16}$ Y. Morita and Y. Hatsugai, Phys. Rev. Lett. 79, 3728 (1997).

${ }^{17}$ W. A. Atkinson, P. J. Hirschfeld, A. H. MacDonald, and K. Ziegler, Phys. Rev. Lett. 85, 3926 (2000).

${ }^{18}$ C. Pépin and P. A. Lee, Phys. Rev. Lett. 81, 2779 (1998).

${ }^{19}$ T. Senthil and M. P. A. Fisher, Phys. Rev. B 60, 6893 (1999).

${ }^{20}$ A. J. Heeger, S. Kivelson, W. P. Su, and J. R. Schrieffer, Rev. Mod. Phys. 60, 781 (1988).

${ }^{21}$ F. Guinea, J. Phys. C 14, 3345 (1981).

${ }^{22}$ F. Guinea and L. Pietronero, Solid State Commun. 49, 269 (1983).

${ }^{23}$ O. Gunnarsson, Rev. Mod. Phys. 69, 575 (1997).

${ }^{24}$ X.-G. Wen, Phys. Rev. B 42, 6623 (1990).

${ }^{25}$ C. L. Kane and M. P. A. Fisher, Phys. Rev. B 46, 15233 (1992).

${ }^{26}$ A. Zunger, Phys. Rev. B 17, 676 (1978).

${ }^{27}$ S. Sorella and E. Tosatti, Europhys. Lett. 19, 699 (1992). 
${ }^{28}$ A. L. Tchougreeff and R. Hoffmann, J. Phys. Chem. 96, 8993 (1992)

${ }^{29}$ F. R. Wagner and M.-B. Lepetit, J. Phys. Chem. 100, 11050 (1996).

${ }^{30}$ M. P. López-Sancho, L. Chico, and M. C. Muñoz (unpublished).

${ }^{31}$ N. B. Hannay, T. H. Geballe, B. T. Mathias, K. Andres, P. Schmidt, and D. MacNair, Phys. Rev. Lett. 14, 255 (1965).

${ }^{32}$ Y. Koike and S. Tanuma, J. Phys. Chem. Solids 41, 1111 (1980).

${ }^{33}$ R. A. Jishi, M. S. Dresselhaus, and A. Chaiken, Phys. Rev. B 44, 10248 (1991); R. A. Jishi and M. S. Dresselhaus, ibid. 45, 12 465 (1992).
${ }^{34}$ L. Edman, B. Sundqvist, E. McRae, and E. Litvin-Staszewska, Phys. Rev. B 57, 6227 (1998).

${ }^{35}$ V. Bayot, L. Piraux, J.-P. Michenaud, J.-P. Issi, M. Lelaurain, and A. Moore, Phys. Rev. B 41, 11770 (1990).

${ }^{36}$ O. E. Andersson, B. L. Prasad, H. Sato, T. Enoki, Y. Hishiyama, Y. Kaburagi, M. Yoshikawa, and S. Bandow, Phys. Rev. B 58, 16387 (1998).

${ }^{37}$ I. Alexandrou, H.-J. Scheibe, C. J. Kiely, A. J. Papworth, G. A. Amaratunga, and B. Schultrich, Phys. Rev. B 60, 10903 (1999).

${ }^{38}$ S. J. Townsend, T. J. Lenosky, D. A. Muller, C. S. Nichols, and V. Elser, Phys. Rev. Lett. 69, 921 (1992).

${ }^{39}$ M.-Z. Huang and W. Y. Ching, Phys. Rev. B 49, 4987 (1994). 